\title{
Zur mathematischen Behandlung der Gegenstromverteilung
}

\author{
Von Peter Karlson und Erich Hecker \\ Aus dem Kaiser-Wilhelm-Institut für Biochemie, Tübingen \\ (Z. Naturforschg. 5 b, 237-246 [1950]; eingegangen am 6. Juli 1950)
}

Die Theorie der einfachen Gegenstromverteilung ist bekannt. Für die komplizierteren Arbeitsweisen, die sich bisher der Berechnung entzogen haben, werden Formeln abgeleitet und für den praktischen Gebrauch in eine Form gebracht, die eine leichte Berechnung der theoretischen Verteilungskurven ermöglicht. Die Anwendungsgebiete der verschiedenen Methoden werden diskutiert und mit experimentellen Ergebnissen verglichen.

$\mathrm{D}$ ie Methode der Gegenstromverteilung hat in jüngster Zeit zunehmende Anwendung gefunden, und es sind von verschiedenén Autoren Apparaturen für die praktische Durchführung angegeben worden ${ }^{1-4}$. Ein besonderer Vorteil der Gegenstromverteilung gegenüber anderen Trennungsmethoden besteht darin, daß sie theoretisch sehr übersichtlich ist: Kennt man den Verteilungskoeffizienten $K$ einer Substanz oder den der Komponenten eines Gemisches, dann kann man das Verteilungsverhalten, d. h. die Menge Substanz in jeder Fraktion, genau vorausberechnen. Für den Grundprozeß haben $\mathrm{Cr}$ a i g und Williams o ${ }^{5}$ die Formel angegeben, und Lie berman ${ }^{6}$ hat ein einfaches Rechenverfahren mitgeteilt (referiert bei $R$ a u e ${ }^{7}{ }^{7}$ ). Es ist auch darauf hingewiesen worden ${ }^{2,8}$, daß die Gegenstromverteilung in verschiedener Weise geleitet werden kann. Für diese Arbeitsweisen, die Methoden der einphasigen, der zweiphasigen und der wechselphasigen Entnahme, haben wir in Anlehnung an Craig ${ }^{5}$ und Lieberman ${ }^{6}$ handliche Formeln entwickelt, die im folgenden zusammengestellt sind.

\section{Der Grundprozeß}

Da die Grundlagen und die Technik der Gegenstromverteilung auch im deutschen Schriftum schon ausführlich behandelt worden sind ${ }^{2,7}$, sei nur ganz

1 L. C. Craig u. O. P os t, Analyt. Chem. 21, 500 [1949]; Ind. Engng. Chem. analyt. Edit. 16, 413 [1944].

2 N.Grubhof er, Chem.-Ing.-Technik 22, 209 [1950].

3 F. W e y g a n d, Chem.-Ing.-Technik 22, 213 [1950].

4 R. Ts chesche u. H. B. König, Chem.-Ing.-Technik 22, 214 [1950].

5 L. C. Craig u. Williamson, J. biol. Chem. 168, 687 [1947].

6 S. O. Li e b e rm a n, J. biol. Chem. 173, 63 [1948].

$7 \mathrm{H}$. R a u en u. W. S t a m m, Chem.-Ing.-Technik 21, 259 [1949].

8 M. T. B u s h u. P. M. D e n s e n, Analyt. Chem. 20, 121 [1948]. kurz darauf eingegangen: Die Substanz wird im ersten Element eines Verteilungsapparates zwischen zwei Lösungsmitteln - z. B. Äther und Wasser verteilt. Nach Schichttrennung wird die Ätherschicht mit neuem Wasser, die wäßrige Phase mit frischem Äther kombiniert (vgl. Abb. 1) und das Gleichgewicht eingestellt. Im nächsten Schritt wird die organische Phase des ersten Elements mit der wäßrigen Phase

\section{Wanderungsrichtung der oberen Phase}
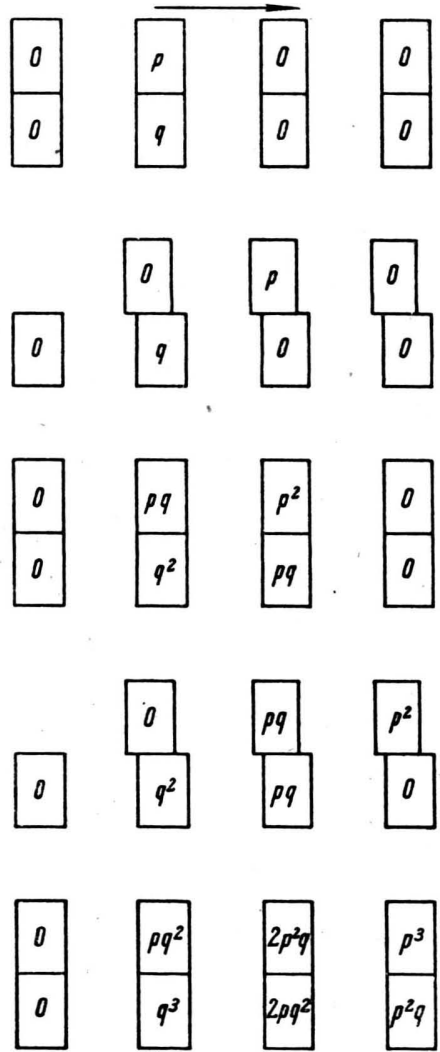

Abb. 1. Schematische Darstellung der ersten Stufen der Gegenstromverteilung. 
des zweiten Elementes kombiniert, während die anderen Phasen mit frischem Äther bzw. Wasser zusammengebracht werden; im folgenden kombiniert jeweils die obere Phase eines Elements mit der unteren des folgenden, im ersten und letzten Element mit neuem Lösungsmittel.

Im Normalfall wird die Verteilung beherrscht vom Nernstschen Satz ${ }^{9}$ :

$$
\frac{c_{1}}{c_{2}}=K \quad \text { oder } \quad \frac{p / V_{\mathrm{p}}}{q / V_{\mathrm{q}}}=K, \quad \frac{p}{q}=K \frac{V_{\mathrm{p}}}{V_{\mathrm{q}}},
$$

$$
\begin{aligned}
& \left(V_{\mathrm{p}}=V_{\mathrm{q}}\right) \text { folgt aus }(1) \\
& \qquad p=\frac{K}{1+K}, \quad q=\frac{1}{1+K} .
\end{aligned}
$$

Wir haben im allgemeinen $p$ und $q$ in die Formeln eingehen lassen; will man nur mit dem Verteilungskoeffizienten $K$ rechnen, dann sind $p$ und $q$ gemäß (3) zu ersetzen.

Mathematisch entspricht der Abb. 1 ein Schema (Abb. 2), das die Ausbreitung des Binoms $(p+q)^{n}$ darstellt. Dieses Schema enthält im Grund schon alle

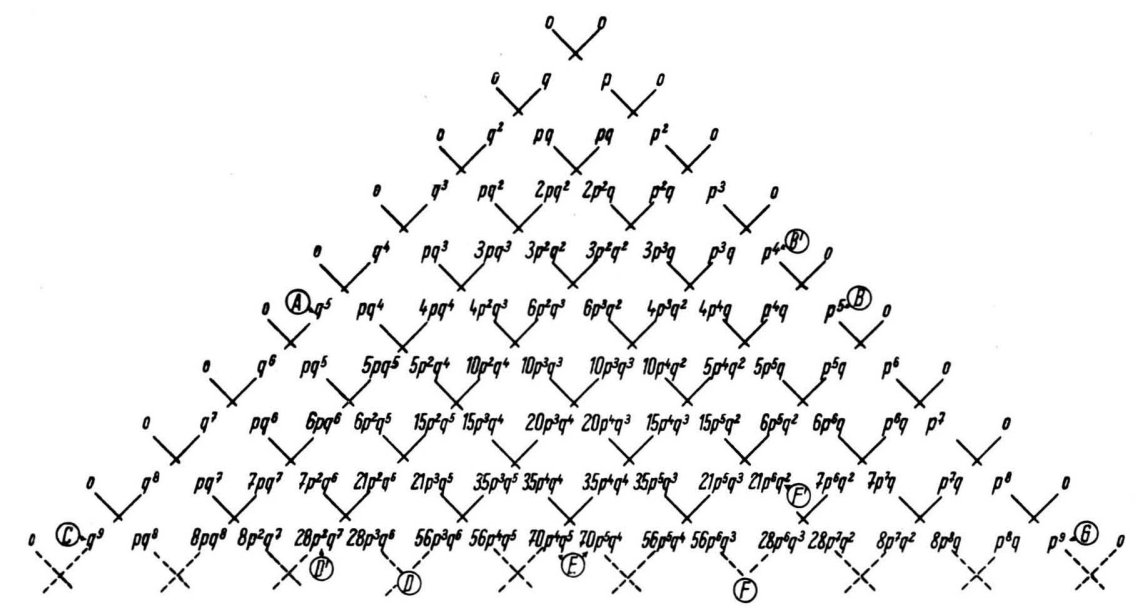

Abb. 2. Mathematisches Schema zur Gegenstromverteilung (Erklärung im Text).

wenn wir $p=\frac{\text { Substanzmenge in der oberen Phase }}{\text { Gesamtsubstanzmenge }}$ und

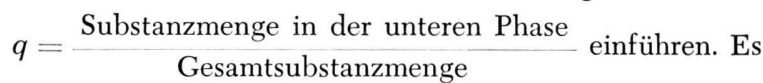
ist also $p+q=1$. - Wie $\mathrm{B}$ u sh und $\mathrm{D}$ en se ${ }^{8}$ gezeigt haben, wird der Trenneffekt für zwei Substanzen A und $\mathrm{B}$ dann am größten, wenn

$$
\frac{V_{\mathrm{p}}}{V_{\mathrm{q}}}=\sqrt{\frac{1}{K_{\mathrm{A}} K_{\mathrm{B}}}}
$$

ist. In der Praxis wird oft mit gleichem Volumen leichter und schwerer Phase gearbeitet. In diesem Falle

9 Der Nernstsche Verteilungssatz ist eine Näherung für den thermodynamisch streng gültigen Ansatz

$$
\begin{aligned}
a_{1} / a_{2}=K \quad \text { oder } \quad c_{1} f_{1} / c_{2} f_{2}=K . \\
(a=\text { Aktivität }, \quad f=\text { Aktivitätskoeffizient })
\end{aligned}
$$

Der Ausdruck $f_{1} / f_{2}$ kann für verdünnte Lösungen organischer Stoffe und für die hier erforderliche Genauigkeit im allgemeinen gleich 1 gesetzt werden. $\mathrm{Ob}$ diese Vereinfachung in einem speziellen Fall zulässig ist, prüft man experimentell durch Ermittlung der Verteilungskoeffizienten bei verschiedenen Konzentrationen, wobei sich im Rahmen der Versuchsfehler Übereinstimmung ergeben muß (vgl. R a u e n ${ }^{7}$ ). Die gegebenen Ableitungen gelten für konzentrationsunabhängiges $K$.
Angaben, die für die mathematische Behandlung der verschiedenen Arbeitsweisen nötig sind.

Die ausgeschriebenen Größen stellen die relativen Anteile Substanz in der Ätherphase und in der Wasserphase (rechts und links unter dem Schnittpunkt) dar, die Diagonalen die weitere Kombination. Die Schnittpunkte sind maßgeblich für die Berechnung der Verteilungskurve, wenn nach C r a ig die Gesamtsubstanz pro Verteilungselement (= Summe Äther- und Wasserschicht) ausgewertet wird. Die Gesamtsumme der Glieder einer Reihe ist $=1$. Die einzelnen Glieder der Reihe (= Frakticnen der Gegenstromverteilung) werden fortlaufend von 0 bis $n$ numeriert, die laufende Nummer wird mit $r$ bezeichnet. Die Richtung der Numerierung ist mit $\mathrm{C}$ r a i g so gewählt, daß Substanzen mit großem $K$, die bevorzugt in die obere Phase gehen, im Schaubild das Maximum rechts (bei hohen Werten für $r$ ) haben. Die gegenläufige Numerierung soll mit $s$ bezeichnet werden. - Die Gesamtzahl der Verteilungsschritte $n$ ist besonders scharf zu definieren. Der erste Verteilungsschritt ist mit der Trennung der Schichten im ersten Verteilungselement und der Kombination mit neuem (leerem) Lösungsmittel erreicht. Nach der Gleichgewichtseinstellung ist immer noch $n=1$, erst mit der Schichttrennung (und Kombination mit frischen Phasen) ist die Verteilungszahl $n=2$ erreicht, wobei jetzt 3 Elemente $(r=0, r=1, r=2)$ 
Substanz enthalten. Allgemein ist also beim Grundproze $\beta$ $n=z-1$, wenn $z$ die Anzahl der benutzten Verteilungselemente ist.

Die relative Substanzmenge in der Fraktion $r$ nach $n$ Verteilungsschritten, $T_{n, r}$ ist gegeben durch

$$
T_{n, r}=\left(\begin{array}{c}
n \\
r
\end{array}\right) p^{r} q^{n-r}=\frac{n !}{r !(n-r) !} p^{r} q^{n-r} .
$$

Craig gibt diese Gl. in der Form

$$
T_{n, r}=\frac{n !}{r !(n-r) !} \frac{K^{r}}{(1+K)^{n}} .
$$

die sich durch Einsetzen von (3) in (4) ergibt.

Gl. (4a) kann zur Berechnung einer beliebigen Fraktion der Verteilung benutzt werden. Für die Ausrechnung der ganzen Verteilungskurve hat L i e $\mathrm{b}$ e r $\mathrm{man}{ }^{6}$ eine elegante Vereinfachung gegeben. Er berechnet logarithmisch jedes Glied aus dem vorhergehenden nach der Gleichung

$$
\log T_{n, r}=\log T_{n, r-1}+\log \frac{n+1-r}{r}+\log K .
$$

Der erste Term der Reihe ist

$$
T_{n, r=0}=-n \log (1+K) .
$$

Die Berechnung selbst geschieht zweckmäßig mit der Addiermaschine, nachdem man $\log \frac{n+1-r}{r}$ für den betreffenden Wert von $n$ tabelliert hat. Dann wird $T_{n, r=0}$ ausgerechnet, in die Maschine gegeben, $\log K$ addiert, $\log _{r=1} \frac{n+1-r}{r}$ addiert, der Wert für $\log T_{n, 1}$

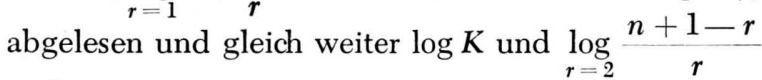
addiert usw. - Tab. 1 gibt in Spalte 2 die Werte für $n=24$ nach Lieberman ${ }^{6}$.

Die Gl. (5) kann in der Form

$$
\log K=\log T_{n, r}-\log T_{n, r-1}-\log \frac{n+1-r}{r}
$$

zur Berechnung von $K$ aus zwei benachbarten Fraktionen im auf- oder absteigenden Kurventeil benutzt werden. Die Bestimmung von $K$ aus $r_{\text {max. }}$ und umgekehrt, für die Lieberma ${ }^{6}$ eine Formel angegeben hat, läßt sich vereinfachen, wenn man berücksichtigt, daß für 2 Fraktionen diesseits und jenseits des Maximums $T_{n, r}=T_{n, r-1}$ ist. Führt man die Gleichung aus, so ergibt sich

$$
K=\frac{r_{\text {max. }}^{\prime}}{n-r_{\text {max. }}^{\prime}+1} .
$$

$r_{\text {max. }}^{\prime}$ ergibt sich aus $r_{\text {max. mit }}$

$$
r_{\text {max. }}^{\prime}=\frac{2 r_{\text {max. }}+1}{2}
$$

exakt für syınmetrische, näherungsweise für die meisten unsymmetrischen Kurven.

Die Formeln (4) und (5) geben die Substanzmenge je Fraktion, also die Summe von Äther- und Wasserphase. Will man aus besonderen Gründen nur eine der beiden Phas n untersuchen, dann gilt für die Substanzmenge in der oberen Phase

$$
T_{n, r}^{\text {oben }}=\left(\begin{array}{c}
n \\
r
\end{array}\right) p^{r+1} q^{n-r}=\frac{n !}{r !(n-r) !} p^{r+1} q^{n-r}
$$

und für die Substanzmenge in der unteren Phase

$$
T_{n, r}^{\mathrm{unten}}=\left(\begin{array}{c}
n \\
r
\end{array}\right) p^{r} q^{n+1-r}=\frac{n !}{r !(n-r) !} p^{r} q^{n+1-r} .
$$

Zur Berechnung kann wieder die Gl.(5) benutzt werden; das Anfangsglied ist für die Oberphase

$$
\log T_{n, r=0}^{\text {oben }}=\log p+n \log q,
$$

für die Unterphase:

$$
\log T_{n, r=0}^{\mathrm{unten}}=(n+1) \log q .
$$

Methode der einphasigen Entnahme

Reicht für die Trennung der Komponenten eines Gemisches die Anzahl der verfügbaren Elemente nicht aus, dann kann man die Verteilung in verschiedener Weise weiterführen. Bei den Verteilungsbatterien von $\mathrm{Craig}{ }^{1}$, von $\mathrm{Grubhofer}{ }^{2}$ und $\mathrm{Tschesche}$ und $\mathrm{König}{ }^{4}$ ist das am einfachsten möglich durch die Methode der einphasigen Entnahme der leichten Phase (im englischen Schrifttum "Single withdrawal“). Dazu nimmt man die Ätherphase am Ende der Verteilungsbatterie fraktionsweise ab und füllt am Anfang neuen Äther nach. Im Schema (Abb. 2) entspricht dieses Vorgehen der Linie C-E-B. Die Folge der Fraktionen setzt sich jetzt zusammen aus einer Grundverteilung nach (4) über $n$ Verteilungsschritte bis zur Fraktion $r=a$ und im zweiten Teil aus den entnommenen Fraktionen, die auf der Diagonale stehen. Diese gehorchen der Gleichung (für $r \geqq a+1)$

$$
\begin{aligned}
T_{n, r}=\left(\begin{array}{c}
n+a-r \\
a
\end{array}\right) & p^{a+1} q^{n-r} \\
& =\frac{(n+a-r) !}{a !(n-r) !} p^{a+1} q^{n-r} .
\end{aligned}
$$


Die Umformung zur Berechnung mit der Addiermaschine in Analogie zu Lieberman ${ }^{6}$ ergibt für $r \geqq a+2$

$\log T_{n, r}=\log T_{n, r-1}+\log \frac{n+1-r}{n+1+a-r}+\log 1 / q$.

Zur Ermittlung der ganzen Verteilungskurve berechnet man zunächst das Anfangsglied nach (5a), dann weiter nach (5) bis zu $r=a$ (bei $z$ Elementen ist

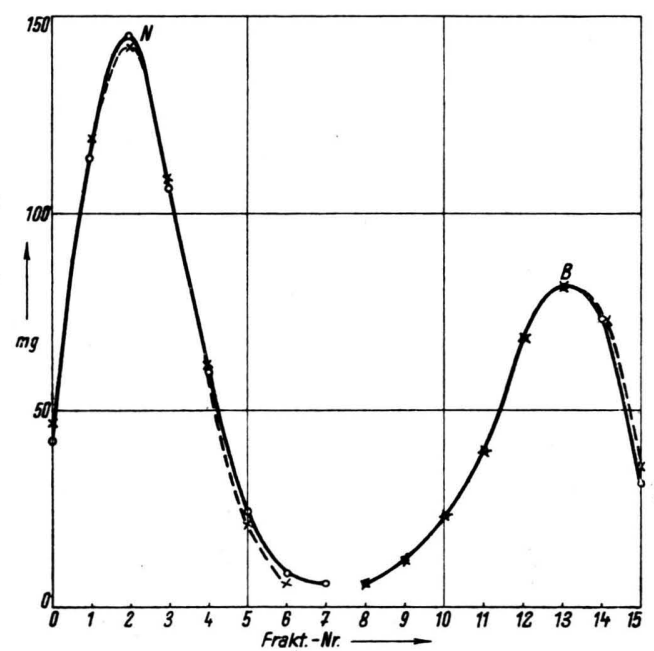

Abb. 3. Verteilung von Nicotinamid (496,3 mg) und Benzamid $(316,5 \mathrm{mg})$ im System Essigester-Wasser nach der Methode der einphasigen Entnahme. Entzogen wurden die Fraktionen 8-15. — gefunden, --- berechnet.

$a=z-1)$. Der Übergang in die Diagonale wird vermittelt durch

$$
\begin{aligned}
\log T_{n, a+1} & =\log T_{n, a}+\log \frac{n-a}{n}+\log K \\
& =\log T_{n, a}+\log \frac{n+1-r}{n+1+a-r}+\log K,
\end{aligned}
$$

d. h. man addiert zu $\log T_{a}$ die beiden oben gegebenen Terme [der erste entstammt der Gl. (9), der zweite der Gl. (5)] und rechnet dann weiter nach (9), wobei man die Werte für $\log \frac{n+1-r}{n+1+a-r}$ vorher tabelliert. Es ist deshalb zweckmäßig, sich bei mehreren Versuchen auf gewisse Werte von $n$ festzulegen, um nicht jeweils neue Tabellen anlegen zu müssen.

Eine mit dieser Methode durchgeführte Verteilung zeigt Abb. 3. Die rechte Kurve ist typisch für die entnommenen Fraktionen.

Will man die schwere Phase entnehmen, dann ergibt sich im Schema die spiegelbildliche Folge AE-G. Zur mathematischen Auswertung können die
Gln. (8) bis (10) dienen, indem man $p$ und $q$ vertauscht und vom anderen Ende her numeriert (hohe $K$-Werte bei niederen Zahlen für $s$ ). Es läßt sich aber auch leicht eine Reihe angeben, die die Berechnung in der üblichen Richtung gestattet:

Die entnommenen Phasen, numeriert von $r=0$ bis $r=a$, sind Glieder der Reihe

$$
\underset{\substack{m, r \\
r \leq a}}{T_{m}}=\left(\begin{array}{c}
m+r \\
r
\end{array}\right) p^{r} q^{m+1}=\frac{(m+r) !}{r ! m !} p^{r} q^{m+1} .
$$

$m$ ist hier und im folgenden die Zahl der Verteilungsschritte, die man ohne Entnahme, d.h. nach dem Grundprozeß durchführt. Bei $z$ Elementen ${ }^{10}$ ist $m=z-1$. - Umgeformt zur logarithmischen Berechnung, ergibt sich für das Anfangsglied

$$
\log T_{m, r=0}=(m+1) \log \mathrm{q}
$$

und für die Reihe

$$
\log T_{r \preceq a}^{m, r}=\log T_{m, r-1}+\log \frac{m+r}{r}+\log p .
$$

Die Werte für $\log \frac{m+r}{r}$ für $m=23$ sind in Tab. 1 aufgeführt. Der Übergang in die Horizontale wird vermittelt durch

$$
\log T_{m, a+1}=\log T_{m, a}+\log \frac{m+r}{r}+\log K,
$$

und die weitere Reihe berechnet sich nach (5), wobei $n=m+a+1$ zu setzen ist.

Der Ansatz (11) kann natürlich auch zur Berechnung der Entnahme der leichten Phasen dienen, wenn man $p$ und $q$ vertauscht und von der anderen Seite her numeriert, beginnend mit der ersten entzogenen Phase $(s=0)$. In dieser Form sollte die Gl. (11) auch die Verhältnisse bei der Verteilungschromatographie beherrschen, denn der Elementarvorgang ist dort der gleiche. Als Voraussetzung ist zu fordern, daß in jedem Volumenelement der Säule das Gleichgewicht völlig erreicht wird, wie in jeder Zelle einer Verteilungsbatterie. Durch geeignete Versuche müßte ferner das Volumenverhältnis von beweglicher und unbeweglicher Phase in einem Volumenelement der Säule und die Zahl der Volumenelemente für eine bestimmte Schichthöhe ermittelt werden. - Auch die übliche Adsorptionschromatographie ließe sich so behandeln, solange die Konzentration der adsorbierten Substanz so gering ist, daß die Konzentrationsabhängigkeit des Adsorptionskoeffizienten zu vernachlässigen ist. Ist das nicht der Fall, so ist die entsprechende Funktion (Langmuiroder Freundlich-Isotherme) einzuführen. Der mathematische Aufwand wird dann aber sehr groß.

10 Arbeitet man mit der Craigschen Verteilungstrommel, dann muß das Element, das zur Entnahme der Fraktionen dient, unbenutzt bleiben und darf nicht mitgerechnet werden. 


\section{Zweiphasige Entnahme}

Dieses von Bush und Dense ${ }^{8}$ in die Praxis eingeführte Verfahren, von den Autoren als „Diamondseparation“ oder „completion of squares“ bezeichnet, führt die Verteilung über den Grundprozeß hinaus, ohne neue Lösungsmittel zuzuführen, und zwar so lange, bis alle leichten Phasen mit allen schweren Phasen im Gleichgewicht gewesen und abgetrennt sind. Im Schema entspricht die Methode den Diagonalen A-E-B. Eine recht umständliche Methode zur Berechnung der theoretischen Kurven wird bereits in der zitierten Arbeit $^{8}$ gegeben; in unserer Schreibweise lautet die Formel

$$
T_{m, r}=\left(\begin{array}{c}
m+r \\
r
\end{array}\right) p^{r} q^{m+1}=\frac{(m+r) !}{m ! r !} p^{r} q^{m+1}
$$

und entspricht damit naturgemäß der ersten Reihe der Unterphasen-Entnahme (11); die maschinelle Rechnung erfolgt nach (12) und (12a). Man kann die Folge der oberen Phasen bei Vertauschung von $p$ und $q$ und entsprechender Numerierung der Fraktionen in derselben Weise berechnen, kann aber auch in der Numerierung fortfahren und die Oberphasen durch die Reihe

$$
\begin{aligned}
\underset{r \geqq a+1}{T_{m, r}} & =\left(\begin{array}{c}
m+2 a+1-r \\
a
\end{array}\right) p^{a+1} q^{m+1+a-r} \\
& =\frac{(m+2 a+1-r) !}{a !(m+a+1-r) !} p^{a+1} q^{m+1+a-r}
\end{aligned}
$$

darstellen. Der Úbergang wird in einfachster Weise durch Addition von $\log K(=\log p / q)$ vermittelt:

$$
\log T_{m, a+1}=\log T_{m, a}+\log K
$$

und die weitere Berechnung geschieht nach

$\underset{r \geqq a+2}{\log T_{m, r}}=\log T_{m, r-1}+\log \frac{m+2+a-r}{m+2+2 a-r}+\log 1 / q$

$$
=\log T_{m, r-1}-\log \frac{m+2+2 a-r}{m+2+a-r}-\log q .
$$

Diese Formeln gelten auch für eine unsymmetrische zweiphasige Entnahme, wobei $m$ die Zahl der Verteilungsschritte ohne Entnahme der Unterphase ist. Experimentell kommt sie dadurch zustande, daß man zunächst nach dem Prinzip der einphasigen Entnahme arbeitet. Man füllt also vorn noch leichte Phase nach, während man am Ende der Batterie schon Fraktionen entnimmt, und läßt dann erst die Batterie „leerlaufen“. Für die symmetrische Arbeitsweise mit einer gleichen Zahl von oberen und unteren Phasen ist $a=m$, und (14a) vereinfacht sich

$$
\underset{r \geqq a+2}{\log T_{m, r}}=\log T_{m, r-1}-\log \frac{3 m+2-r}{2 m+2-r}-\log q .
$$

Die Werte von $\log \frac{3 m+2-r}{2 m+2-r}$ sind symmetrisch zu den Werten von $\log \frac{m+r}{r}$, so daß man hier nur eine Tabelle benötigt, die einmal abwärts, einmal aufwärts gelesen wird.

Abb. 4 zeigt den Typ der Verteilungskurven, die sich nach dieser Methode ergeben, im Vergleich mit der Grundverteilung. Die Kurven sind für eine Substanz mit $K=2,25$ berechnet.

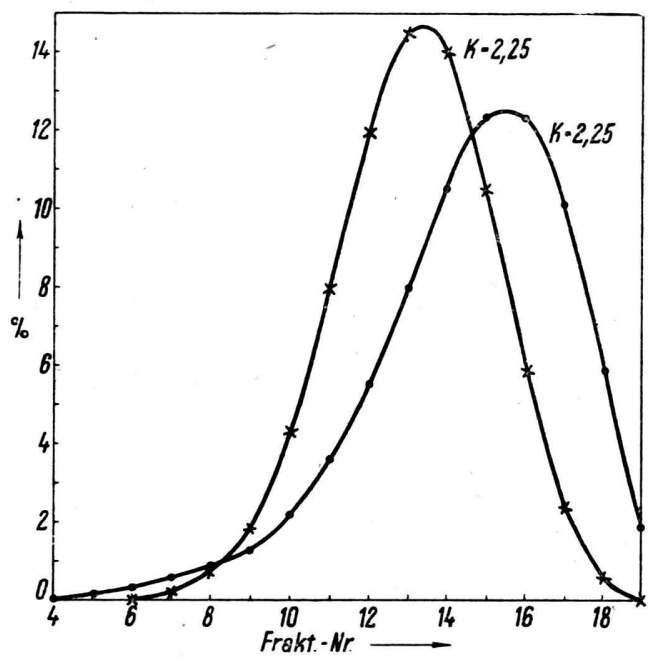

Abb. 4. Theoretische Verteilungskurve nach der Methode der zweiphasigen Entnahme $(\bullet-\bullet)$ im Vergleich mit dem Grundprozeß $(\times \stackrel{-}{-})$.

\section{Methodeder wechselphasigen Entnahme}

Mit diesem Namen haben wir die Arbeitsweise belegt, die von $\mathrm{Cr}$ aig als "alternate withdrawal " bezeichnet wird. Sie geht in der Weise vor sich, daß nach Beendigung des $m$-stufigen Grundprozesses immer abwechselnd einmal Ätherphase, einmal wässerige Schicht entnommen und ergänzt wird. Die übrigen Elemente des Verteilungsapparates bleiben dabei stets mit beiden Phasen gefüllt.

Auf die experimentellen Schwierigkeiten bei der Durchführung dieses Verfahrens in der Verteilungsbatterie hat $\mathrm{Gru} \mathrm{b} \mathrm{h}$ of e ${ }^{2}$ hingewiesen; bei Verwendung der Craigschen Trommel oder des Apparates von $\mathrm{Ts} \mathrm{che} \mathrm{s} \mathrm{che} 4$ bzw. W e y g a n $\mathbf{d}^{3}$ dürfte es jedoch leicht durchzuführen sein.

Die mathematische Behandlung des Verfahrens ist schwierig und war bisher nicht möglich. Im Schema (Abb. 2) entsprechen die entnommenen Fraktionen den Senkrechten von A nach $\mathrm{D}^{\prime}$ und von $\mathrm{B}^{\prime}$ nach $\mathrm{F}^{\prime}$. Diese 
Linien treffen immer abwechselnd auf Größen, die zur oberen Phase und solche, die zur unteren Phase gehören; es ist also nur jede zweite Größe ein Glied der Reihe der entnommenen Fraktionen, denn diese Reihe besteht ja ausschließlich aus entnommenen oberen bzw. unteren Phasen. - Die außen stehenden Teile des Schemas werden durch die Entnahme abgeschnitten und liefern keine Substanz mehr nach innen nach. Diese Tatsache zwingt zur Einführung von Korrekturgliedern, die die Formeln erheblich komplizieren. Deshalb ist es auch einfacher, von beiden Ästen her zu rechnen, d. h. die entnommenen organischen Phasen gesondert zu behandeln.

Die unten gegebenen Formeln sind unter folgenden Voraussetzungen abgeleitet:

1. Die erste entnommene Fraktion ist eine leichte Phase;

2. es werden gleichviel leichte und schwere Phasen entnommen;

3. die Zahl der auf einer Seite entnommenen Fraktionen ist nicht größer als die Zahl der vorhandenen Verteilungselemente.

Der Ansatz für die entnommenen wäßrigen Phasen lautet dann

$$
\begin{aligned}
& T_{m, r}=\left[\left(\begin{array}{c}
m+2 r+1 \\
r
\end{array}\right)-\left(\begin{array}{c}
m+2 r+1 \\
r-1
\end{array}\right)\right] p^{r} q^{m+2+r} \\
= & {\left[\frac{(m+1+2 r) !}{r !(m+1+r) !}-\frac{(m+1+2 r) !}{(r-1) !(m+r+2) !}\right] p^{r} q^{m+2+r} . }
\end{aligned}
$$

Die beiden Glieder der Klammer lassen sich auf einen Nenner bringen:

$$
T_{m, r}=\frac{(m+2)(m+1+2 r) !}{r !(m+r+2) !} p^{r} q^{m+2+r}
$$

und für logarithmische Berechnung umformen:

$$
\begin{aligned}
& \log T_{r \leq a} \\
& =\log T_{m, r-1}+\log \frac{(m+2 r+1)(m+2 r)}{r(m+r+2)}+\log p q,
\end{aligned}
$$

wobei das Anfangsglied

ist.

$$
T_{m, r=0}=(m+2) \log q
$$

In ganz entsprechender Weise läßt sich die Folge der entnommenen oberen Phasen, die von $s=0$ bis $s=a$ numeriert werden, darstellen:

$$
\begin{aligned}
& \underset{\underset{\underline{m}}{m, s}}{T_{a}}=\left[\left(\begin{array}{c}
m+2 s \\
s
\end{array}\right)-\left(\begin{array}{c}
m+2 s \\
s-1
\end{array}\right)\right] p^{m+1+s} q^{s} \\
& =\frac{(m+1)(m+2 s) !}{s !(m+s+1) !} p^{m+1+s} q^{s} .
\end{aligned}
$$

Die maschinelle Berechnung geschieht nach

$\log T_{m, s}$

$=\log T_{m, s-1}+\log \frac{(m+2 s)(m+2 s-1)}{s(m+s+1)}+\log p q$

mit dem Anfangsterm

$$
\log T_{m, s=0}=(m+1) \log p .
$$

Für $m$ und $a$ sind dieselben Werte wie bei der $r$-Reihe einzusetzen $\left(m=z-1\right.$; vgl. auch $\left.{ }^{10}\right)$. Tab. 1 gibt in der 4. und 5. Spalte die erforderlichen Werte für $m=23$.

Der Mittelteil der Verteilung, die Horizontale im Schema (D-F), läßt sich nicht als Ausschnitt aus einer Grundverteilung nach (4) darstellen, wie $\mathrm{Grubh}$ of e ${ }^{2}$ annimmt; auch hier müssen Korrekturglieder eingeführt werden. Der vollständige Ansatz lautet

$$
\begin{aligned}
& T_{n, r}=T_{n, r}^{\prime}-t_{n, r}^{\prime}-t^{\prime \prime}{ }_{n, r} \\
& =\left(\begin{array}{c}
n \\
r
\end{array}\right) p^{r} q^{n-r}-\left(\begin{array}{c}
n \\
2 a-r
\end{array}\right) p^{r} q^{n-r}-\left(\begin{array}{c}
n \\
r-m-1
\end{array}\right) p^{r} q^{n-r},
\end{aligned}
$$

wobei $n=m+2 a+2$ und $a+1 \leqq r \leqq m+a+1$ ist.

Hier ist es nicht möglich, alle drei Glieder auf einen Nenner zu bringen und zur logarithmischen Rechnung mittels Addiermaschine umzuformen. Es muß jede Reihe für sich berechnet werden. Die Grundreihe

$$
T_{n, r}^{\prime}=\left(\begin{array}{c}
n \\
r
\end{array}\right) p^{r} q^{n-r}
$$

ermittelt man nach (4) und (5) mit dem Anfangsglied

$$
\begin{gathered}
\log {T^{\prime}}_{n, a+1}=\log n !-\log (a+1) ! \\
-\log (n-a-1) !+(a+1) \log p+(n-a-1) \log q
\end{gathered}
$$

oder aus der zuletzt entzogenen Fraktion [Gl. (18), $r=a$ ] durch das Übergangsglied

$\log T_{n, r=a+1}^{\prime}$

$=\log T_{m, r=a}+\log \frac{(m+2 a+2)(m+a+2)}{(m+2)(a+1)}+\log K$.

Das erste Korrekturglied

$$
\begin{aligned}
t_{n, r}^{\prime}=\left(\begin{array}{c}
n \\
2 a-r
\end{array}\right) p^{r} q^{n-r} & \\
& =\frac{n !}{(2 a-r) !(m+r+2) !} p^{r} q^{n-r}
\end{aligned}
$$




\begin{tabular}{|c|c|c|c|c|c|}
\hline $\begin{array}{c}\text { Frakt.- } \\
\text { Nr. } \\
r\end{array}$ & $\begin{array}{l}\text { Grundmethode } \\
\log \frac{n+1-r}{r} \\
\text { Gl. (5) } n=24\end{array}$ & $\begin{array}{l}\text { Einphasige Ent- } \\
\text { nahme der unteren } \\
\text { Phase } \\
\log \frac{m+r}{r} \\
\text { Gl. (12) } m=23\end{array}$ & $\begin{array}{c}\text { Wechselphasige } \\
\text { Entnahme der unteren } \\
\text { Phase } \\
\log \frac{(m+2 r+1)(m+2 r)}{r(m+r+2)} \\
\text { Gl. (18) } m=23\end{array}$ & $\begin{array}{c}\text { Wechselphasige } \\
\text { Entnahme der oberen } \\
\text { Phase } \\
\log \frac{(m+2 s)(m+2 s-1)}{s(m+s+1)} \\
\text { Gl. }(20) m=23\end{array}$ & $\begin{array}{c}\text { Frakt.- } \\
\text { Nr. } \\
s\end{array}$ \\
\hline $\begin{array}{r}1 \\
2 \\
3 \\
4 \\
5 \\
6 \\
7 \\
8 \\
9 \\
10 \\
11 \\
12 \\
13 \\
14 \\
15 \\
16 \\
17 \\
18 \\
19 \\
20 \\
21 \\
22 \\
23 \\
24\end{array}$ & $\begin{array}{l}1,3802 \\
1,0607 \\
0,8653 \\
0,7202 \\
0,6021 \\
0,5006 \\
0,4102 \\
0,3274 \\
0,2499 \\
0,1761 \\
0,1047 \\
0,0348 \\
9,9652-10 \\
9,8953-10 \\
9,8239-10 \\
9,7501-10 \\
9,6726-10 \\
9,5898-10 \\
9,4994-10 \\
9,3979-10 \\
9,2798-10 \\
9,1347-10 \\
8,9393-10 \\
8,6198-10\end{array}$ & $\begin{array}{l}1,3802 \\
1,0969 \\
0,9379 \\
0,8293 \\
0,7482 \\
0,6842 \\
0,6320 \\
0,5883 \\
0,5509 \\
0,5185 \\
0,4901 \\
0,4649 \\
0,4424 \\
0,4221 \\
0,4037 \\
0,3870 \\
0,3717 \\
0,3575 \\
0,3444 \\
0,3325 \\
0,3213 \\
0,3108 \\
0,3010 \\
0,2919\end{array}$ & $\begin{array}{l}1,3979 \\
1,1461 \\
1,0153 \\
0,9321 \\
0,8739 \\
0,8309 \\
0,7978 \\
0,7716 \\
0,7503 \\
0,7329 \\
0,7183 \\
0,7060 \\
0,6955 \\
0,6864 \\
0,6785 \\
0,6717 \\
0,6657 \\
0,6603 \\
0,5554 \\
0,6513 \\
0,6474 \\
0,6441 \\
0,6410\end{array}$ & $\begin{array}{l}1,3802 \\
1,1303 \\
1,0011 \\
0,9192 \\
0,8622 \\
0,8203 \\
0,7880 \\
0,7627 \\
0,7422 \\
0,7252 \\
0,7112 \\
0,6994 \\
0,6893 \\
0,6807 \\
0,6731 \\
0,6666 \\
0,6609 \\
0,6558 \\
0,6512 \\
0,6472 \\
0,6437 \\
0,6404 \\
0,6375\end{array}$ & $\begin{array}{r}1 \\
2 \\
3 \\
4 \\
5 \\
6 \\
7 \\
8 \\
9 \\
10 \\
11 \\
12 \\
13 \\
14 \\
15 \\
16 \\
17 \\
18 \\
19 \\
20 \\
21 \\
22 \\
23\end{array}$ \\
\hline
\end{tabular}

Tab. 1. Laufende Terme zur Berechnung der theoretischen Verteilungskurven.

läßt sich logarithmisch berechnen nach der Gleichung

$$
\log t_{n, r}^{\prime}=\log t_{n, r-1}^{\prime}-\log \frac{m+2+r}{2 a+1-r}-\log 1 / K
$$

für $a+m+1 \geqq r \geqq a+2$.

Das Anfangsglied, $\log t_{n, a+1}^{\prime}$, kann entweder aus (23) berechnet werden, indem man $r=a+1$ einsetzt:

$\log t_{n, r=a+1}^{\prime}=\log n !-\log (a-1) !-\log (m+a+3) !$

$$
+(a+1) \log p+(m+a+1) \log q
$$

oder aus der Reihe (18), die für die Senkrechte gilt, durch die Übergangsbeziehung

$\log t_{n, r=a+1}^{\prime}$

$$
=\log T_{m, r=a}+\log \frac{(m+2 a+2) a}{(m+2)(m+a+3)}+\log K .
$$

Der Ansatz für das zweite Korrekturglied lautet

$$
\begin{aligned}
t_{n, r}^{\prime \prime} & =\left(\begin{array}{c}
n \\
r-m-1
\end{array}\right) p^{r} q^{n-r} \\
& =\frac{n !}{(r-m-1) !(n+m+1-r) !} p^{r} q^{n-r} .
\end{aligned}
$$

Für die praktische Rechnung wird umgeformt zu

$$
\log t^{\prime \prime}{ }_{n, r}=\log t^{\prime \prime}{ }_{n, r-1}+\log \frac{n+m+2-r}{r-m-1}+\log K .
$$

Die Reihe beginnt bei $r=m+1$. Das Anfangsglied ergibt sich zu

$$
\log t^{\prime \prime}{ }_{n, r=m+1}=(m+1) \log p+(n-m-1) \log q .
$$

Wir haben den laufenden Term in Gl. (24) als Subtrahenden gegeben, weil die Werte von $\log \frac{m+2+r}{2 a+1-r}$ und $\log \frac{n+m+2-r}{r-m-1}[\mathrm{Gl} .(28)]$ gleichzeitig Glieder der Reihe $\log \frac{n+1-r}{r}$ darstellen, die für die Berechnung der Grundreihe [Gl. (22)] nötig sind. Man braucht also für die Horizontale nur eine Tabelle, die aber für $r_{T^{\prime}}, r_{t^{\prime}}$ und $r_{t^{\prime \prime}}$ in verschiedener Weise gelesen wird. Die Reihen für die Korrekturglieder erlöschen, wenn der Nenner in $\frac{m+2+r}{2 a+1-r}$ [Gl. (24)] Null, in $\frac{n+m+2-r}{r-m-1}$ [Gl. (28)] negativ wird; die folgenden Glieder der Grundreihe bleiben dann ohne Korrektur oder bekommen nur einen Korrekturterm anstatt deren zwei. - In den allermeisten Fällen werden 
die Korrekturglieder $t^{\prime}{ }_{n, r}$ und $t^{\prime \prime}{ }_{n, r}$ nur bei einigen wenigen Fraktionen in der Nähe des Übergangs zu den entnommenen Fraktionen größer als $1 \%$ von $T^{\prime}{ }_{n, r}$ sein; und zwar ist dies für $t^{\prime}{ }_{n, r}$ der Fall, wenn

$$
r>a+\frac{1}{\log \left(1+\frac{m+2}{a+1}\right)} \quad \text { für } r \leqq 2 a,
$$

für $t^{\prime \prime}{ }_{n, r}$, wenn

$r>m+a+1,5-\frac{1}{\log \left(1+\frac{m+1}{a+1}\right)} \quad$ für $\quad r \leqq 2 a$

ist. Man kann an Hand dieser Ungleichungen leicht abschätzen, für welche Werte von $r$ man die Korrekturreihen berücksichtigen muß und dann das erste fragliche

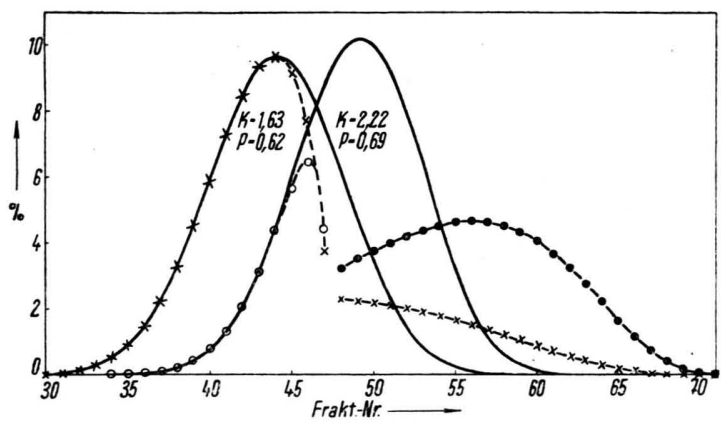

Abb. 5. Berechnete Kurven für die Verteilung zweier Substanzen mit $p=0,62$ und $p=0,69$ nach dem Grundprozeß (ausgezogene Kurve) und der wechselphasigen Entnahme (gestrichelte Kurve). Die für das Substanzgemisch zu erwartende Verteilung ergibt sich als Summe der Einzelkurven.

Glied jeder Reihe mit Hilfe von Gl. (23) bzw. (27) ausrechnen.

Die Formeln (16) bis (20) werden unbrauchbar, sobald man mehr Fraktionen auf einer Seite entnimmt, als Elemente auf dem Verteilungsapparat sind. Wird $a+1>z$, dann tauchen auch in den vertikalen Reihen, d. h. bei den entnommenen Fraktionen, zweite Korrekturglieder auf. Auf die Berechnung dieser Glieder wurde verzichtet, weil sie in der Praxis selten vorkommen. Meist wird man sie bis $a+1 \approx 3 / 2 z$ vernachlässigen können.

Entzieht man wechselweise zwei organische und eine wäßrige Phase, dann ergeben sich Reihen, die sich gleichfalls ausrechnen ließen. Wir haben diese sehr speziellen Arbeitsweisen aber nicht in den Kreis der Betrachtungen einbezogen.

Zwei vollständig durchgerechnete Kurven der wechselphasigen Entnahme sind in Abb. 5 dargestellt, und zwar für Stoffe mit $p=0,62$ und $p=0,69$, deren Maxima in der Nähe der Übergangsstelle liegen. Charakteristisch ist das scharfe, zusammengedrängte Maximum am Ende des Mittelteils; auch ist zu beachten, daß eine einheitliche Substanz zwei Maxima geben kann. Zum Vergleich sind die Grundverteilungskurven mit eingezeichnet.

Anwendungsbereiche der verschiedenen Methoden und Vergleich mit experimentellen Ergebnissen

An Hand der berechneten Kurven in Abb. 3 bis 7 sei die Leistungsfähigkeit der verschiedenen Methoden kritisch betrachtet. Für analytische Arbeiten wird man in erster Linie die Grundmethode heranziehen. Die Kurven verlaufen hier am steilsten, und eine Verunreinigung der Substanz wird sich schon bei sehr ähnlichem Verteilungskoeffizienten durch eine Abweichung vom berechneten Wert bemerkbar machen. Zur Prüfung einer experimentellen Kurve auf Einheitlichkeit der verteilten Substanz berechnet man $K$ nach ( $5 \mathrm{~b}$ ) aus je zwei benachbarten Werten im aufsteigenden und absteigenden Kurventeil ${ }^{11}$; die $K$ Werte müssen gleich sein. - Úberdies liefert die Grundmethode die reinsten Präparate (allerdings mit geringer Ausbeute).

Die verschiedenen Entnahmeverfahren sollen vor allem dazu dienen, mit einer geringen Zahl von Verteilungselementen einen ähnlichen Trennungseffekt zu erzielen wie mit einer Grundverteilung in einem größeren Apparat. Wenn diese Methoden im folgenden mit einer Grundverteilung gleicher Fraktionenzahl verglichen werden, dann ist zu berücksichtigen, daß mit dem verwendeten Apparat diese Grundverteilung nicht in einem Arbeitsgang gewonnen werden kann.

Die Methode der einphasigen Entnahme hat ihre Vorteile auf experimentellem Gebiet. Sie ist einfach durchzuführen; schon mit 8 Verteilungselementen gelang uns eine quantitative Trennung von Nicotinamid und Benzamid (vgl. Abb. 3); die berechneten und gefundenen Kurven stimmen gut überein. Entnimmt man sehr viele Fraktionen, dann kann die Substanz restlos in das leichtere Lösungsmittel übergeführt werden (allerdings werden die Kurven bei $K<1$ sehr breit). Das ist besonders dann von Vorteil, wenn man z. B. mit Pufferlösung als schwerer Phase arbeitet, weil darin die Gehaltsbestimmung oft schwierig ist. Es ist jedoch zu erwägen, ob man dann

11 Diese Möglichkeit besteht natürlich ebenso bei den anderen Methoden mittels Gl. (12) bzw. (18) oder (20). Liegt eine Mischung aus nur zwei Substanzen vor, dann lassen sich grundsätzlich aus vier Kurvenpunkten die Verteilungskoeffizienten und die Mengen der beiden Substanzen berechnen; der mathematische Aufwand wird aber sehr groß. 
nicht zur unsymmetrischen zweiphasigen Entnahme greift, die dasselbe leistet.

Die zweiphasige Entnahme eignet sich besonders für präparative Arbeiten; sie kann sogar der Grundverteilung mit gleicher Zahl von Fraktionen überlegen sein. Eine berechnete Verteilung für ein Sub-

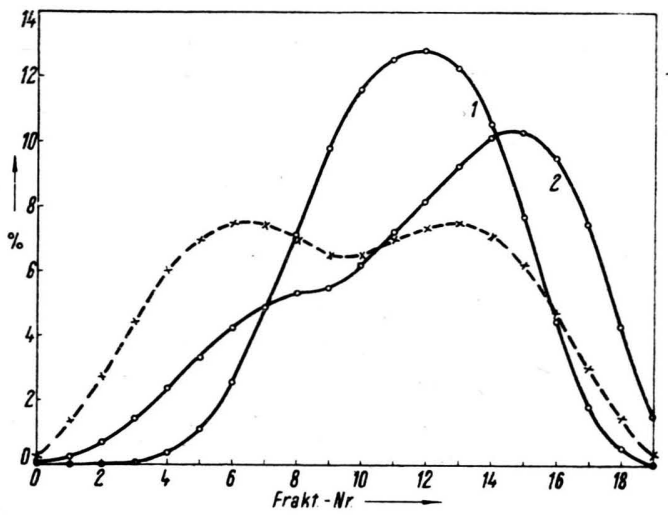

Abb. 6. Vergleich der Trenneffekte bei der zweiphasigen Entnahme (Kurve 2) und beim Grundprozeß (Kurve 1) für ein Gemisch aus $50 \% \mathrm{~A}$ mit $K=1,00$ und $50 \% \mathrm{~B}$ mit $K=2,25$ (vgl. auch Abb. 4 und Tab. 2).

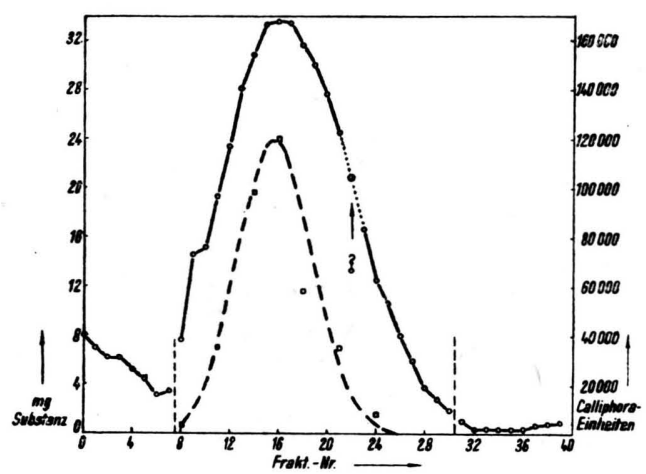

Abb. 7. Experimentelle Gegenstromverteilung eines Insektenextrakts (॰- $\bigcirc$ Gewichtskurve; --- theoret. Kurve für $K=0,676$; $\square$ exper. gefundene Wirksamkeit).

stanzgemisch aus $50 \%$ A mit $K=1,00$ und $50 \%$ B mit $K=2,25$ zeigt Abb. 6. Teilt man die Kurve in drei Abschnitte und berechnet die darin enthaltene Substanzmenge, so ergibt sich folgendes Bild (Tab. 2):

Man erkennt, daß zur Darstellung sehr reiner Substanz die Grundmethode besser geeignet ist, während bei der Gewinnung größerer Mengen nicht so sehr reiner Substanz die zweiphasige Entnahme den Vorzug verdient: Im gewählten Beispiel sinkt der Anteil der Mischfraktion (Nr. 7-13) von $72 \%$ auf $43,5 \%$. - Geht man vom Volumenverhältnis $1: 1 \mathrm{ab}$ und paßt es den beiden Verteilungskoeffizienten nach (2) an, so erhält man einen

\begin{tabular}{|c|c|c|c|c|}
\hline \multirow[b]{2}{*}{$\begin{array}{l}\text { Frakt.- } \\
\text { Nr. }\end{array}$} & \multicolumn{2}{|c|}{ Grundmethode } & \multicolumn{2}{|c|}{$\begin{array}{c}\text { Zweiphasige } \\
\text { Entnahme }\end{array}$} \\
\hline & $\begin{array}{l}\text { Gesamt- } \\
\text { substanz } \\
\text { in } \%\end{array}$ & $\begin{array}{l}\text { Reinheits- } \\
\text { grad } \\
\text { bezogen } \\
\text { auf A } \%\end{array}$ & $\begin{array}{l}\text { Gesamt- } \\
\text { substanz } \\
\text { in } \%\end{array}$ & $\begin{array}{l}\text { Reinheits- } \\
\text { grad } \\
\text { bezogen } \\
\text { auf A } \%\end{array}$ \\
\hline $0-6$ & 4,2 & 99,3 & 12,7 & 95,6 \\
\hline $7-13$ & 72,0 & 60,0 & & \\
\hline $14-19$ & 23,8 & 7,1 & 43,8 & 18,0 \\
\hline
\end{tabular}

Tab. 2. Vergleich der Grundmethode mit der zweiphasigen Entnahme. Verteilung eines Substanzgemisches von 50\% A mit $K=1,00$ und $50 \%$ B mit $K=2,25$ (vgl. auch Abb. 6).

\begin{tabular}{|c|c|c|c|c|}
\hline \multirow{2}{*}{$\begin{array}{c}\text { Frakt.- } \\
\text { Nr. }\end{array}$} & \multicolumn{2}{|c|}{ Grundmethode } & \multicolumn{2}{|c|}{$\begin{array}{c}\text { Wechselphasige } \\
\text { Entnahme }\end{array}$} \\
\cline { 2 - 5 } & $\begin{array}{c}\text { Substanz } \\
\text { in } \%\end{array}$ & $\begin{array}{c}\text { Reinheits- } \\
\text { grad } \\
\text { bezogen } \\
\text { auf A in } \%\end{array}$ & $\begin{array}{c}\text { Substanz } \\
\text { in } \%\end{array}$ & $\begin{array}{c}\text { Reinheits- } \\
\text { grad } \\
\text { bezogen } \\
\text { auf A in } \%\end{array}$ \\
\hline $30-44$ & 33,3 & 81,0 & 33,3 & 81,1 \\
$45-47$ & 23,9 & 54,0 & 18,6 & 54,0 \\
$47-71$ & 42,7 & 23,5 & 48,0 & 26,2 \\
\hline
\end{tabular}

Tab. 3. Vergleich der Grundmethode mit der wechselphasigen Entnahme nach Abb. 5 für $50 \%$ A mit $K=1,63$ und $50 \% \mathrm{~B}$ mit $K=2,22$.

optimalen Trenneffekt, der für die zweiphasige Entnahme durch die gestrichelte Kurve dargestellt ist.

Besonders wertvoll ist die zweiphasige Entnahme dann, wenn sehr große Substanzmengen verarbeitet werden sollen, wenn man also zu Scheidetrichtern greifen muß 8.

Die wechselphasige Entnahme entspricht weitgehend einer Grundverteilung, wie Abb. 5 zeigt. Zwar macht sich bei den entnommenen Fraktionen eine unangenehme Verschleppung der Substanz bemerkbar; teilt man jedoch die Kurven in drei Abschnitte und vergleicht wie oben, dann zeigen sich nur geringe Unterschiede (Tab. 3).

Die wechselphasige Entnahme scheint deshalb besonders geeignet für Substanzen bzw. Gemische mit einem Verteilungskoeffizienten, der nicht $\mathrm{zu}$ sehr von 1 abweicht. Sie verbindet die Vorteile der Grundmethode mit der Möglichkeit, durch Auffangen vieler Fraktionen die Verteilung sehr weit zu führen. Ein Beispiel aus der Laborpraxis mag das noch erläutern: Abb. 7 zeigt eine Verteilung eines Extrakts aus Insektenmaterial, den wir mit dem Ziel der Isolierung des Puparisierungshormons ${ }^{12}$ hergestellt und in bezug auf diesen Wirkstoff schon weitgehend angereichert haben. Die Verteilung wurde im System EssigesterButanol 4:1/Wasser über 39 Verteilungsschritte geführt. Das Hauptmaximum enthält ein Stoffgemisch,

12 Erich B e cker, Biol. Zbl. 61, 360 [1941]. 
in welchem der gesuchte Wirkstoff durch seine biologische Aktivität nachweisbar ist. Die Auswertung mehrerer Fraktionen führte zur Aufstellung einer Wirksamkeitskurve, die trotz der Schwankungen des biologischen Tests recht gut einer Kurve mit $K=0,676$ folgt. (Zur Berechnung von $K$ wurden die Werte von $r=11$ und $r=14$ herangezogen.) Im Gebiet um $r=26$ befand sich eine krystallisierende Begleitsubstanz, die auf diese Weise abgetrennt wurde.

Für die Überprüfung der Rechnungen danken wir Hrn. $\mathrm{R}$ i e c k e r t vom Mathematischen Institut der Universität Tübingen.

\title{
Über das sogenannte „६-Hexachlor-cyclohexan“ II $^{1}$
}

\author{
Von R. Riemschneider und G. Ottmann* \\ Aus dem Chemischen Institut der Freien Universität Berlin-Dahlem** \\ (Z. Naturforschg. 5 b, 246-250 [1950]; eingegangen am 2. Mai 1950)
}

\begin{abstract}
Das während der Chlorierung von Cyclohexan in beträchtlicher Menge entstehende 1.2.4.5Tetrachlor-cyclohexan vom Schmp. 173-174 ${ }^{\circ}$ läßt sich nicht als ein Zwischenprodukt bei der Bildung von " $\zeta$-Hexachlor-cyclohexan" ansprechen; seine möglichst weitgehende Abtrennung ist vorteilhaft, wenn ",-Hexachlor-cyclohexan“ aus Cyclohexan-Chlorierungsprodukten isoliert werden soll.

,$\zeta$-Hexachlor-cyclohexan“ besitzt nur eine sehr geringe insektizide Wirkung.
\end{abstract}

$\mathrm{W}$ ie der eine von uns vor kurzem gezeigt hat ${ }^{2}$, ist das bei der Chlorierung von Cyclohexan entstehende Hexachlorcyclohexan $(\mathrm{HCH})$ vom Schmp. $146^{\circ} 3$, das sogenannte ,ל-Hexachlor-cyclohexan“" , bisher nur wenig untersucht worden; seine Konstitution wurde noch nicht sichergestellt. Die 1947 von Bastiansen und Mitarbb. ${ }^{4}$ vertretene Ansicht, daß dieses Isomere zur Reihe der stereoisomeren 1.2.3.4.5.6-Hexachlor-cyclohexane gehört, teilen wir nicht ${ }^{5}$.

Da sich das sogenannte „ל-Hexachlor-cyclohexan“ nach der von uns zunächst ausgearbeiteten Herstellungsvorschrift ${ }^{2}$ nur in verhältnismäßig geringer Ausbeute erhalten läßt und Versuche über das chemische Verhalten sowie die Aufklärung der Konstitution des

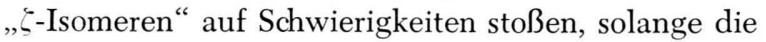

* Anschrift der Verfasser: Berlin-Nikolassee, Hohenzollernplatz 1 .

** Vorliegende Arbeit ist z. Tl. im Physiologisch-chemischen Institut der Humboldt-Universität Berlin ausgeführt worden.

1 Mitteilung I (R. Riemschneider, Über das sogenannte „६-Hexachlor-cyclohexan“ I, Mitt. Physiolog.chem. Inst. R 33, März 1949) ist nur auszugsweise veröffentlicht worden, und zwar in den Abschnitten „Die sechs wichtigsten Hexachlorcyclohexane“ und „Die Chlorierung des Cyclohexans“ in der Schrift „Zur Kenntnis der Kontakt-Insektizide II“, 9. Beih., 1. Erg.Bd. zur „Pharmazie“ 1949, S. 737, 738, 746, 751, 752 und 754 (vgl. auch „Besprechungen“, dieses Heft, S. 289).
Verbindung nicht in größerer Menge und leicht zugänglich ist, haben wir die Chlorierung des Cyclohexans erneut untersucht. Gleichzeitig hofften wir, aus derartigen Versuchen (insbesondere durch getrennte Weiterchlorierung der bei der Halogenierung von Cyclohexan zunächst gebildeten isolierbaren niederen Chlorierungsprodukte bekannter Konstitution sowie durch ihre Aufarbeitung und ihre Prüfung auf evtl. gebildetes „“') gewisse Anhaltspunkte über die Konstitution des Hexachlor-cyclohexans vom Schmp. $146^{\circ} \mathrm{zu}$ erhalten, die zusammen mit den bei der Einwirkung von Alkali bzw. Zinkstaub auf das Isomere

2 R. Riemschneider, 9. Beih., 1. Erg.Bd. zur „Pharmazie“, S. 738, 751. - Die älteren Untersucher haben die Herstellung des Hexachlorcyclohexans vom Schmp. $146^{\circ}$ nur sehr ungenau beschrieben und machten keine Ausbeutungsangaben.

3 Sämtliche Temperaturangaben in Celsius-Graden.

4 Die für das bei $146^{\circ}$ schmelzende Hexachlorcyclohexan von O. B a s ti a n s e n und Mitarbb. (Acta chem. scand. 1, 683 [1947]), vorgeschlagene Bezeichnung „รHexachlor-cyclohexan" sollte richt oder nur in Anführungszeichen gebraucht werden, da die für Hexachlorcyclohexane eingeführte griechische Buchstabenbezeichnung nur die Reihenfolge ihrer Entdeckung anzeigt, das sogenannte „ $\zeta$-Isomere" jedoch bereits vor $\gamma, \delta$ und $\varepsilon$, also als drittes Isomeres, entdeckt worden ist ${ }^{2}$. Vgl. auch Anz. Schädlingskunde 23, 62 [1950].

5 Begründung in Mitteilung $\mathrm{I}^{1}$ und in der in Fußnote 2 zitierten Arbeit. - N a chtrag : vgl. auch Research 2 248 [1949]. 